\title{
LA REFLEXIVIZACIÓN COMO RECURSO DE PERSPECTIVIZACIÓN EN LA EXPRESIÓN DE MOVIMIENTO EN ESPAÑOL
}

\begin{abstract}
Resumen. El uso de la construcción reflexiva para denotar un movimiento direccional no causativo suele explicarse en términos aspectuales. La presente contribución tiene por objetivo mostrar que, en realidad, la diferencia con la construcción no reflexiva no atañe a la estructuración temporal interna del evento como tal, sino que afecta a la representación de la participación de la entidad sujeto. Adoptando el marco de la lingüística cognitiva, se postula que la marca reflexiva simboliza el acceso mental al despliegue de energía en el participante desde una perspectiva interna que capta la dimensión experiencial de su relación cambiante con el entorno espacial, salvando al mismo tiempo la visión de conjunto por la desmultiplicación del perfil de la entidad sujeto. Además de verificar esta hipótesis, el cotejo de usos de ir e irse y de salir y salirse permite separar el significado construccional de efectos de sentido discursivo-pragmáticos variables.
\end{abstract}

Palabras clave: movimiento, construcción reflexiva, perspectiva, significado construccional.

\section{Planteamiento}

Esta contribución se centra en el perfil particular que, en español, las construcciones reflexivas de tipo 'medio' dan a la expresión de movimiento en comparación con las intransitivas no pronominales. Las hipótesis existentes apelan primordialmente a diferencias aspectuales. En Sánchez López (2002: 115 ss.) se pasa revista a las hipótesis propuestas desde marcos teóricos diversos. Más allá de diferencias terminológicas, en particular entre aproximaciones generativas y cognitivas, los análisis parecen estar de acuerdo en considerar la reflexivización no argumental fundamentalmente como una marca de perfectividad. Si bien es cierto que en muchos contextos se pueden observar efectos de sentido parecidos a la superación de un límite, no es siempre el caso que se demarque un

\footnotetext{
* KU Leuven.
} 
punto clave en un determinado desarrollo espacio-temporal. No es raro, además, que para hacer referencia a un determinado cruce de umbral se pueda acudir alternativamente a un predicado no reflexivo. ¿Cuál será entonces la aportación de la marca reflexiva?

La necesidad de buscar una aclaración alternativa se plantea sobre todo cuando con la construcción reflexiva no se expresa un movimiento fáctico, sino uno que se puede llamar metafórico, abstracto o ficticio, carente de proyección espacio-temporal y, por ende, de fases. Para que haya perfectividad en el sentido clásico, debe ser posible distinguir entre un punto inicial y un punto final, cada uno con un antes y un después, que a su vez pueden dar paso a interpretaciones ingresivas o resultativas. Resulta difícil ver cómo se puede aplicar el concepto de realización o de transición puntual a entidades estacionarias o a entidades abstractas, a menos de disociar la noción de perfectividad del vector temporal. Por razones de espacio, sin embargo, solo será posible abordar aquí la expresión de desplazamientos fácticos de tipo direccional.

La hipótesis, que se presenta aquí por primera vez, parte de la idea de que no yerra la intuición de que la reflexivización de alguna manera influye en la interpretación 'aspectual'. Esta, sin embargo, no se traduce a nivel de la estructuración interna del evento o proceso tal como corresponde al significado léxico del verbo, sino que afecta a la representación imagística de la participación de la entidad sujeto. Dicho de otro modo, la modificación afecta a la estructuración gestáltica de la involucración de la entidad sujeto. Por eso, tal vez sea más acertado definir la diferencia entre la construcción intransitiva no reflexiva y la reflexiva en términos de perspectiva(ción), en el caso de movimientos fácticos, y de subjetivización con movimientos ficticios. Así se puede hacer justicia a la opción entre dos tipos de representaciones 'imagísticas', a saber, una escenificación global, establecida desde un punto de vista distante, sin enfoque particular en la vinculación del sujeto al predicado, o, al contrario, una captación desde cerca que invita, por así decirlo, a aplicar un deslizador de zoom, descubriendo cómo en la entidad sujeto 'se encarna' un movimiento o cambio, sea este fáctico o ficticio.

Epistemológicamente, la presente propuesta se apoya en el punto de partida siguiente. En la codificación de la escena se otorga la primacía al conceptualizador, es decir, a la persona que determina el significado de un enunciado. Al procesar las experiencias perceptivas convirtiéndolas en representaciones mentales, el conceptualizador interpreta las sensaciones que lo conectan con el entorno añadiendo un significado a los estímulos sensoriales. Por el sesgo cognitivo hacia el dinamismo («cognitive bias 
towards dynamism», Talmy, 2000: 171), el conceptualizador tiende a proyectar su propia movilidad física y mental en su visión del entorno, privilegiando la dinamicidad y la activación a la estatividad y la inacción. De ahí que sea práctica común utilizar lenguaje de movimiento y cambio para describir escenas estáticas y situaciones estables.

La información proveniente del exterior se amolda así a la subjetividad del conceptualizador, o sea, a la posición particular que es la suya en un determinado momento. La perspectivación procede de su receptividad o permeabilidad al impacto de una entidad sobre su propio 'espacio mental'. Los espacios mentales se definen como las estructuras representacionales que los hablantes constituyen al reflexionar y hablar. Aunque de naturaleza inestable y local, se estructuran según dominios cognitivos (cf. Fauconnier, 1985). En la conceptualización de movimiento y de cambio se entrelazan las dimensiones del espacio y del tiempo; en el dominio del movimiento predomina el espacio y en el dominio del cambio prevalece el tiempo.

Además de las capacidades cognitivas e imaginativas, interviene en el proceso interpretativo el saber enciclopédico adquirido, en particular el conocimiento relativo a la función o rol que puede desempeñar la entidad descrita, sea cual sea su naturaleza, y la manera en que puede destacar en el contexto. En la línea de la lingüística cognitiva, se parte de la idea de que las formas lingüísticas explotan y representan las diferencias de acceso a la información en términos de procesamiento mental, conectando la información al punto de vista subjetivo de un conceptualizador. Se reconoce que el hablante puede acceder a informaciones similares o idénticas procesándolas de forma diferente según la perspectiva que adopte y la visión del mundo que maneje en un determinado momento. De ahí que se considere que el significado corresponde a la estructuración conceptual (traducción del término inglés construal, cf. Verhagen, 2007: 48; Langacker, 2008: 4), es decir, la manera en que una situación está representada: además de las propiedades objetivas de lo denotado, es crucial la forma en que el conceptualizador opta por (re)presentarla.

De acuerdo con el lema langackeriano «semantics is conceptualization», consideramos que el uso del lenguaje (y su significado) no está determinado por la realidad objetiva y externa sino que está relacionado con la manera de percibir esa realidad, es decir, con la conceptualización que proyectamos sobre ella. De ahí que cualquier expresión lingüística siempre implique una relación entre la situación conceptualizada (objeto de conceptualización) y algún conceptualizador (sujeto de conceptualización). En el caso que nos interesa aquí, el objeto de conceptualización es la representación de un 
evento de movimiento o cambio. El sujeto de conceptualización, es decir, el conceptualizador, la persona que determina o resuelve el significado atribuido a una expresión, está situado en la base de la construcción; con el término base se hace referencia a la situación comunicativa, los participantes y sus circunstancias. Langacker (1987: 128) califica esta relación de relación de «construal», concepto que traducimos por estructuración conceptual ${ }^{1}$ :

I will say that the speaker (or hearer), by choosing appropriate focal 'settings' and structuring a scene in a specific manner, establishes a construal relationship between himself and the scene so structured. The construal relationship therefore holds between the conceptualizer of a linguistic predication and the conceptualization that constitutes this predication.

Ahora bien, toda codificación lingüística viene impregnada de una cierta dosis de subjetividad. La selección que se opera entre diversas formulaciones posibles para denotar un movimiento o un cambio de estado será reveladora del perfilamiento que el conceptualizador proyecta en la entidad móvil o cambiante. No es el mero reflejo de lo directa e inmediatamente accesible sensorialmente, sino que procede también de su particular marco de referencia, su mundo e imaginación. Significa que es una interpretación de la relación entre el predicado y la entidad sujeto. En la subjetivización del movimiento o cambio puede verse un proceso regular de perspectivación conceptual. La perspectiva(ción) desempeña un papel central en la interfaz entre gramática y discurso. Por perspectiva se entiende la relación entre la posición del conceptualizador-papel asumible no solo por el hablante-autor sino también por el interlocutor-lector -y el contenido de una expresión. $\mathrm{Al}$ abordar este concepto relacional general suelen entrar en consideración varias conceptos, entre otros: subjetividad, subjetivización, punto de vista, posicionamiento, orientación, horizonte, aspecto, (puesta de) relieve, primer vs segundo plano... son algunos de los conceptos que se emplean en estudios interesados en la interfaz entre gramática y discurso.

A partir de estas consideraciones generales, se procura mostrar a continuación que la marca reflexiva añade una dimensión que no es simplemente asimilable a una cuestión de aspecto. Se ilustrará cómo la perspectiva interviene en la expresión de movimiento fáctico. Por razones

${ }^{1}$ Se observa una estrecha correspondencia entre esta relación de estructuración conceptual y el concepto de perspectiva o «viewing arrangement» tal como lo define Langacker (2008: 73): «A viewing arrangement is the overall relationship between the viewers and the situation being viewed». Para más información, véase Langacker (1987: 128-132, 2008: 73-78). 
de espacio las referencias bibliográficas quedan limitadas a un mínimo y las pocas líneas dedicadas a la expresión de movimiento y cambio ficticios se relegan al final.

\section{Construcciones reflexivas que expresan movimiento}

Como objeto de conceptualización, los movimientos fácticos se definen esencialmente por la extensión y orientación de una trayectoria combinadas con el vector del tiempo concebido. La distancia recorrida por unidad de tiempo define la velocidad de ejecución del movimiento. La noción de avance se compone del acercamiento a una nueva posición o ubicación combinado con su correlato, el movimiento de alejamiento o abandono: la toma de conciencia de la nueva posición o estado es una proyección fuera del instante insecable en que se sitúa la materialización del proceso expresado por el verbo. No hay relación unívoca entre los lexemas verbales y el perfilamiento de parámetros adicionales, como son la intencionalidad, el control, la brusquedad. Su interpretación tampoco depende de cómo se construya el verbo, reflexivamente o no (ir(se), marchar(se), salir(se), venir(se), etc.), sino que se infiere del contexto.

Sin más contexto, puede decirse que en las dos variantes presentadas en (1) se dan por identificados el participante humano y el espacioorigen, y se toma como punto de referencia el espacio-meta. La realidad extralingüística puede ser la misma. Tanto en la construcción reflexiva como en la no reflexiva el verbo de movimiento direccional invita a trazar mentalmente una trayectoria, cuya extensión va necesariamente delimitada por ambos lados, es decir, que además del punto de origen incluye el punto en que se detiene el movimiento, independientemente de que este se conciba como meta final del recorrido o no.

[Va / Se va] a la cocina

Entre la forma no marcada y la marcada mediante el clítico reflexivo media, según la hipótesis de trabajo, una diferencia de perspectiva. La variante no reflexiva describe un tipo de desplazamiento desde una posición de observación externa, es decir, sin adoptar el punto de vista interno al participante. La construcción reflexiva, en cambio, señala la presencia de un perceptor cercano, empático, que se proyecta en el participante, o se muestra por lo menos capaz de ponerse en el lugar del que se mueve. La idea de que el clítico se simboliza el acceso mental al progresivo despliegue de energía en el participante implica que el 
movimiento se concibe como una acción particular, no rutinaria. La atención prestada a la fuerza motriz que anima al participante realza al mismo tiempo el interés por las modalidades y circunstancias en que ocurre.

Todo movimiento es descomponible en tres fases constitutivas: la transición inicial de un estado a la activación orientada a un nuevo estado, el desarrollo del movimiento, y la transición final que desemboca en el nuevo estado. Como el punto de arranque es la condición sine qua non para que haya movimiento, será lógicamente el primer punto de interés. De ahí que la marca reflexiva tienda a interpretarse como una invitación a fijar la atención en la transición de inmovilidad a movilidad. Esto probablemente explica que varios autores hayan insistido en que con irse la meta es «irrelevante» y que se destaque que el participante «abandona un sitio para dirigirse a otro» (cf. Maldonado, 1997: 167 y ss.). Según la visión aspectual, lo que hace el clítico se «es ubicar el punto específico en que se da el cambio de estado» (Maldonado, 1997: 169). Por atractivo que sea este planteamiento, si hay que interpretarlo en términos aspectuales, parecería implicar que se recorta el componente puntual logro que representa la transición inicial, es decir, el momento del abandono del estado que corresponde al punto de origen, relegando a un segundo plano el subevento central de la realización así como el estado resultante una vez alcanzada la meta.

Ahora bien, incluso si se puede hacer abstracción de la finalización, parece difícil, tratándose de un verbo direccional, prescindir de una representación, sea mínima, de la orientación. Desde el punto de vista del ente que se mueve, el movimiento va necesariamente de su aquí a un allí. La integración de anterioridad, interioridad y posterioridad opera en la entidad sujeto, trascendiendo la distinción aspectual entre fase ingresiva, cursiva y resultativa, que se expresa por otros medios, en particular, por auxiliares aspectuales.

Independientemente de la cuestión de saber si, léxicamente, la transición expresada por el verbo se clasifica por defecto como puntual o no, es decir, como logro o más bien como realización en términos vendlerianos, la media reflexiva centra la atención en la dinamización de la estructuración conceptual del movimiento tal como se encarna en la entidad sujeto. Su movilidad adaptativa induce una visión desmultiplicada de su relación cambiante con el entorno. Funciona, pues, como un recurso para combinar espacio y tiempo: la idea de que cada fracción del movimiento es disociable de las demás es traducible en la representación mental de sendas réplicas o imágenes duplicadas que, concatenadas, dejan un rastro a lo largo del recorrido. 
Esta conceptualización no equivale a una repartición en fases, sea una subdivisión bifásica, trifásica o polifásica. En términos de visualización, la construcción se-REFL de interpretación media produce un efecto óptico que recuerda al fenómeno de la diplopía: la imagen de la posición hacia la cual la entidad sujeto hace el movimiento no se mantiene constante sino que se dobla instantáneamente de una nueva imagen, la de la posición que el mismo movimiento le hace abandonar para alcanzar o ir alcanzando otra posición. El morfema se incita, pues, a enlazar iterativamente retrospección y prospección, y esto hasta la total recreación imaginaria del movimiento. Guardar en la mente a cada paso actualizador tanto la mirada retrospectiva como la prospectiva proporciona una conceptualización conjunta de fases contiguas del movimiento que es comparable, mutatis mutandis, a la representación pictórica con que los pintores futuristas captaban la sensación de movimiento: descomponían el movimiento para superponer posiciones sucesivas reuniéndolas en un solo plano para que resaltara la trayectoria de los gestos. Este procedimiento permite dar una visión fluida y a la vez secuencializada por combinar fracciones temporales concatenadas, o sea, no separadas entre ellas. En ciertos contextos, esta visión sinóptica no parte simplemente de la posición ocupada en el instante inicial $t_{0^{\prime}}$ sino que puede incluir fracciones anteriores $\left(t_{-n}\right)$ de la misma manera que comprende la progresión $\left(t_{+n}\right)$ hacia la meta: .... $t_{-5}-t_{-4}-t_{-3}-t_{-2}-t_{-1}-$ $t_{0}-t_{1}-t_{2}-t_{3}-t_{4}-t_{5}-t_{6}-t_{7} \ldots$ La extensión del ámbito espacial abarcado varía en función del predicado, de la entidad sujeto y del contexto más amplio. El efecto de desmultiplicación así obtenido da la impresión de asistir desde cerca al movimiento, viviéndolo desde dentro, reconstruyendo su progresivo desenvolvimiento.

En muchos contextos se puede optar por una forma u otra. Sin embargo, la escenificación que las dos variantes contribuyen a construir es de índole diferente. Compárese, por ejemplo, (2) y (3)². En (2), la cercanía subjetiva marcada por el clítico reflexivo particulariza la actuación de Paco como parte de una escena más compleja. El contacto mental con su situación se extiende más allá del momento de partida: el movimiento orientado se vincula con la interlocución con Javi, por un lado, y la interacción con Tere, por otro. El enfoque específico se apoya tanto en el discurso directo que precede, como en la construcción de gerundio que sigue. No se habría obtenido la misma cohesión contextual mediante la construcción no marcada. En cambio, esta última encaja mejor en (3): del contexto se desprende que el indicativo presente recibe una interpretación habitual. Se infiere, además,

${ }^{2}$ Los ejemplos provienen del Corpus de Referencia del Español Actual (CREA), más en particular del dominio de la ficción, y limitado a España. 
que entre las secuencias descritas en la subordinada temporal y la principal hay una relación de causa a efecto: una vez acabado su trabajo, le espera indefectiblemente la preparación de la cena. Parafraseando, se puede decir que Renato espera, para acudir a ver al viejo, a que su esposa deje su trabajo y vaya a la cocina. En la observación de lo que hace ella se refleja pues la perspectiva externa de Renato. En la imagen global, los respectivos quehaceres de los esposos se asimilan fácilmente a un principio de reparto de las tareas domésticas. Para saber si esto corresponde a un acuerdo libremente asumido o revela, al contrario, un distanciamiento entre ellos, se necesita más contexto. Con la forma alternativa, se va a la cocina, al contrario, se habría adoptado la perspectiva experiencial de la mujer, movida por una dinámica interna; su desplazamiento habría resaltado como una iniciativa personal, no como la simple continuación de las actividades diarias, en continuidad con su actividad laboral.

(2) Javi ¡Pues claro, tío, perfectamente! ¡Y que sea doble la ración! (Paco se va a la cocina y empieza a trajinar. Dando a Tere unas palmaditas amistosas en la espalda) (CREA, C. Resino, Pop y patatas fritas, 1991).

(3) Cuando ella deja su trabajo y va a la cocina, Renato acude a ver al viejo. Se lo encuentra tumbado, siempre con la luz apagada (CRE A, J. L. Sampedro, La sonrisa etrusca, (1985) 1995).

Tanto el origen como la meta pueden quedar sobreentendidos al ser contextualmente recuperables, o sea, que siguen presentes en la base. Se puede prescindir de complementación locativa tanto con ir (4) como con irse (5). Además del carácter auto-causativo, compartido con las demás personas, la primera persona destaca en ambos casos la autoconciencia del yo que se proyecta en un movimiento direccional. La diferente extensión cronológica y cronométrica no es atribuible al tipo de construcción, sino que viene circunstancialmente determinada: en (4) la inminencia se calcula en segundos, en (5) más bien en horas o días; en (4) se evoca un trayecto corto que se recorre en nada de tiempo; en (5), en cambio, se sobreentiende una distancia significativa cuyo recorrido toma su tiempo.

(4) - Y ahora, mi amor, ven aquí.

- Voy.

- Vamos a encender la luna en la terraza, que ya es hora (CREA, L. Beccaria, La luna en Jorge, 2001).

(5) Hablaron él y Amparito, con las caras muy juntas para que no se oyera la charla.

- Me voy.

- Dónde.

- A la capital.

- ¿Con quién? (CREA, R. del Pozo, La novia, 1995) 
Voy (4) se interpreta convencionalmente como acto de habla con valor de compromiso. En este binomio llamada-respuesta la reacción es rutinaria: ante la facilidad de ejecución del desplazamiento requerido, no se hace hincapié en la dinámica de fuerzas en que el enunciador pudiera verse envuelto. Se trata simplemente de registrar y aceptar una meta preestablecida que se puede dar automáticamente por alcanzada, ya que pertenece al mismo espacio de interlocución. Decir me voy produciría el efecto contrario, a saber, la prefiguración experiencial de una separación en lugar de un reencuentro.

En (5), en cambio, sería incongruente que el personaje anunciara su decisión de marcharse a la capital por un simple voy. No es un desplazamiento sin consecuencias sino un viaje que representa un cambio de vida, una ruptura con lo familiar, un salto hacia lo desconocido. Al marcar el impacto que el yo puede experimentar anticipativamente, la reflexivización invita a una simulación mental de la dinámica de fuerzas que puede operar en él, tanto retrospectiva como prospectivamente. Pero lo hace solo al nivel esquemático de la construcción: cuántos impulsos y frenos, tanto psíquicos como físicos, le puedan afectar se deja a la imaginación.

La transición puntual denotada por el verbo de logro salir(se) se orienta de dentro a fuera. El espacio de origen se concibe como una interioridad asimilable a un contenedor, o sea, a un espacio cerrado, mientras que la meta, la exterioridad, aparece como un espacio abierto, no delimitado. Tanto salir como salirse operan una bipartición del espacio. Salir ofrece una instantánea del paso que lleva la figura de un lado de la línea de demarcación al otro lado. Cuando no se precisa el origen, se induce por defecto que se trata de su espacio-universo propio -la casa o el lugar donde está habitualmente- y adonde volverá.

Así, en expresiones como salir a la calle (6) o salir de paseo se suele sobreentender salir de casa. El uso absoluto, sin ninguna complementación locativa tampoco concede importancia a los lugares por donde pueda andar la persona, señala simplemente su ausencia (7). Mientras que en (6) y (7) se da por contado que en algún momento volverá, en (8) la sucesión de eventos sugiere que el abandono del lugar es irreversible. Los tres ejemplos tienen en común, sin embargo, que no invitan a enfocar la modalidad del acceso al espacio abierto ni el contraste con su previa estancia en el espacio cerrado, sea este su hábitat ((6)-(7)) o un lugar de tránsito (8).

(6) Me pregunto qué sentirá uno cuando sale a la calle un viernes por la noche (CREA, E. Lindo, La ley de la selva, 1996). 
(7) - La señorita Beatriz no está...

- ¿Ha salido?

Cecilia, que apenas era un susto perpetuamente cosido a un delantal, asintió.

- ¿Sabes cuándo volverá? (CRE A, C. Ruiz Zafón, La sombra del viento, [2001] 2003)

(8) Chalán sale de su escondite, corre hasta el Opel y abre de un tirón la puerta delantera (CREA, J. Ribera, La sangre de mi hermano, 1988).

La reflexivización, en cambio, adopta la perspectiva del participante, amplificando su «ventana de atención» (Talmy, 2000: 257 y ss.): mientras su campo de visión se extiende hacia el horizonte, guarda, por así decirlo, la capacidad de mirar en el retrovisor. El cotejo entre la previa relación con el entorno y la nueva permite evaluar cómo le afecta la traslación o cambio de lugar. El clítico reflexivo opera como una lente de conversión gran angular. Aunque el grado de apertura puede variar según el contexto, el principio del travelling perceptual hacia atrás y hacia delante puede articularse como sigue: al igual que el punto $t_{0}$ de la transición aparece como el 'pasado' inmediato respecto del instante postcedente $t_{+1}, \mathrm{y} \mathrm{t}_{+1}$ a su vez como el antecedente de $t_{+2}$ ' etc., en el punto $t_{0}$ se ve también el 'futuro' del instante precedente $t_{-1}$, $y$ en $t_{-1}$ el postcedente de $t_{-2}$, etc.

Así se puede empáticamente tomar en cuenta la situación del 92 participante como se desenvuelve en el espacio pos-transicional y en el espacio pre-transicional. Desde esta perspectiva interna, la localización del participante en los instantes de $t_{-n}$ a $t_{-1}$ arroja luz sobre su paso, en $t_{0^{\prime}}$ a la nueva relación con el entorno en el instante $t_{0+1}$. El contraste que se percibe entre las dos ubicaciones conlleva fácilmente una dimensión evaluativa que puede resultar positiva (9) o negativa (10).

(9) ¿Qué película célebre detesta?

- Quizá, Casablanca.

- ¿Se sale del cine? ¿De qué película se salió la última vez?

- Sí, me salgo. Generalmente de películas francesas.

- Algo especial que le sucedió en un cine.

- Son sitios muy aburridos (CREA, El Mundo, 13.04.1996).

(10) A las dos de la mañana, un turismo se salió de la carretera en una de las cuestas de la Dehesa de la Villa y acabó empotrándose contra un árbol. El conductor, que estaba borracho, se había hecho un lío con los pies y había pisado el acelerador en vez del freno (CREA, A. Grandes, Los aires difíciles, 2002).

En cuanto a la dimensión pragmática, el alcance explicativo de la noción de contra-expectativa es muy relativo. En un mismo contexto pueden coexistir expectativas divergentes según cuál sea el nivel intra o extradiscursivo considerado. El participante, posibles testigos de la escena, y otros personajes de la historia, por un lado y, por otro, el 
conceptualizador-narrador responsable de la formulación y los lectoresreceptores del enunciado no tienen por qué proyectarse necesariamente en el mismo tipo de guion. Esta variabilidad se observa tanto para las construcciones no reflexivas como para las reflexivas.

Por el contexto de (9) (la última vez, generalmente) se entiende que el entrevistado encuentra tan natural abandonar un cine como otros se van de paseo ((6), (7)) o buscan escapar (8). Su comportamiento tampoco le sorprenderá al entrevistador si es un conocido suyo, aunque en su artículo de periódico no lo deje trasparentar, y entre los lectores podrá haber reacciones tan variadas como ante el predicado no reflexivo. Además, si en (7) la ausencia de la señora puede ir en contra de las expectativas del visitante, probablemente no les causa sorpresa a sus cohabitantes. Tanto en (7) como en (8), la posición del narrador parece opaca respecto del carácter más o menos esperable de la situación.

No cabe duda, en cambio, de que en (10) la pérdida del control del vehículo no es deseada por sus ocupantes ni por nadie; en las circunstancias descritas, sin embargo, el riesgo de accidente era, sin duda, conocido y reconocido tanto por los implicados como por los observadores, entre ellos la autora-narradora y los lectores. Este ejemplo muestra que no queda excluida la mención de factores externos que, sin ser la causa directa del evento, facilitan su ocurrencia y permiten explicarlo (embriaguez del conductor, oscuridad, zona forestal).

De los ejemplos (6)-(10) se desprende que no es cierto que el se «energético» «comprime el ejercicio de energía y elimina de la escena consideraciones sobre el conjunto de circunstancias implicadas en la evolución de un evento» (Maldonado, 1997: 172). Por el carácter puntual del evento tipo logro, el traspaso del límite entre interioridad y exterioridad puede, en efecto, considerarse veloz y aparecer como abrupto e instantáneo, que la construcción esté reflexivizada o no. Esto no significa, sin embargo, que la conceptualización de la escena, en particular de la involucración del participante presente necesariamente las mismas características.

Tanto en (8) como en (10) no cabe duda acerca de la rapidez de ejecución, y es altamente plausible su brusquedad. Sin embargo, incluso para el accidente de coche, presentado en (10), dar el paso inferencial suplementario hacia la «accidentalidad», definida como el hecho de que «el acaecer del evento vaya en contra de las expectativas naturales del conceptualizador» (Maldonado, 1997: 173) resulta problemático. Es posible, en efecto, sustituir la secuencia coordinada (y acabó empotrándose contra un árbol) por una frase prospectiva introducida por para (para empotrarse contra un árbol) que se interpreta como una consecuencia natural, 
no como una finalidad; el conceptualizador puede, pues, considerar como previsible un acontecimiento de por sí excepcional como es una súbita salida de pista.

En (9), finalmente, resulta difícil proyectar la imagen de un acto instantáneo, brusco, rápido o llamativo. Nada sugiere que la persona desista repentina o accidentalmente de visionar una película ni que salga del cine de forma ostentativa. Del contexto se desprende, al contrario, el carácter habitual, voluntario y asumido, de un comportamiento motivado por preferencias personales. De hecho, el enunciado es compatible con una cláusula genuinamente final, lo que refuerza el carácter razonado de su aserción. Además, el propio yo-conceptualizador especifica qué tipo de producción es más susceptible de aburrirle (películas francesas). Además de subrayar su capacidad anticipativa, esto muestra que se rige por criterios consistentes, de modo que, desde su perspectiva, su actuación no tiene nada de sorprendente. Tildarla de inesperada implica juzgar que va en contra de expectativas vigentes. Esto, sin embargo, supone manejar tácitamente alguna norma sociocultural, lo cual lleva el proceso inferencial más allá de lo que dice el texto.

\section{Reflexión final}

La hipótesis presentada en estas páginas aborda la reflexivización como una operación de estructuración conceptual cuyo significado gramatical no es asimilable al de una marca aspectual. Como aclaración alternativa se postula que la noción de perspectiva interna, por oposición a la perspectiva externa de la construcción no reflexiva, realza la interconexión entre el perfil dinámico de la entidad sujeto y la experiencia espaciotemporal denotada por el predicado. La construcción no reflexiva no destaca particularmente esta interrelación, sino que simplemente categoriza el tipo de movimiento que se atribuye a la entidad sujeto. La construcción reflexiva, en cambio, refuerza su envolvimiento: se enfoca en la interfaz y convergencia entre la dinámica del proceso y la del participante que lo encarna. La marca reflexiva estimula el acceso perceptual a la relación cambiante del participante con el entorno mientras que, por simulación mental, se construye al mismo tiempo la visión de conjunto a través de una imagen desmultiplicada del perfil de la entidad sujeto. La aportación conceptual de la reflexivización consiste, pues, en facilitar una visión a la vez fragmentada y unificada del movimiento. 
El alcance de esta propuesta se ha podido comprobar en algunos ejemplos contrastados de ir e irse, por un lado, y salir y salirse, por otro. No se niega que, a partir de indicaciones contextuales y de conocimientos del mundo, el envolvimiento de la entidad sujeto puede a veces interpretarse adicionalmente como intencional o no, veloz o no, brusco o no, sorprendente o no, accidental o no, etc. Pero es información que se deriva del contexto y cuya validez puede variar en función del punto de vista del conceptualizador. Además, la ocasional activación de tales efectos de sentido discursivo-pragmáticos no es exclusiva de la construcción reflexiva sino que se da también en la no reflexiva. Dicho de otro modo, no pertenece como tal al significado esquemático de la construcción reflexiva.

En un trabajo futuro queda por ampliar el análisis. Primero, conviene cotejar las construcciones reflexiva y no reflexiva de un mayor número de verbos. Luego, hay que extender la comparación a verbos de raíz léxica diferente (por ejemplo, ir / dirigirse). También se impone la extensión del dominio del movimiento al de cambios de estado (por ejemplo, crecer / incrementarse). Finalmente, conviene estudiar la expresión de movimientos y cambios ficticios (por ejemplo, serpentear / deslizarse, véase Delbecque, 2015).

\section{Bibliografía}

DELBECQUE, N. (2015). «Variable aspectual coercion in Spanish fictive motion expressions», en E. BARRAJÓN LÓPEZ, J. L. CIFUENTES HONRUBIA y S. RODRÍGUEZ ROSIQUE (eds.), Verb Classes and Aspect. Amsterdam: J. Benjamins, 153-184.

FAUCONNIER, G. (1985). Mental spaces: Aspects of meaning construction in natural language. Cambridge, MA: MIT Press.

LANGACKER, R. W. (1987). Foundations of Cognitive Grammar. Volume 1: Theoretical Prerequisites. Stanford, CA: Stanford University Press.

LANGACKER, R. W. (2008). Cognitive Grammar. A Basic Introduction. Oxford: Oxford University Press.

MALDONADO, R. (1997). «Dos trayectos, un sentido. Rutas conceptuales de la accidentalidad», en R. BARRIGA VILLANUEVA y P. MARTÍN BUTRAGUEÑO (eds.), Varia Lingüística y Literaria. 50 Años del Centro de Estudios Lingüísticos y Literarios, I. Lingüística. México D. F.: El Colegio de México, 165-189. 
REAL ACADEMIA ESPAÑOLA. Corpus de Referencia del Español Actual (CREA) [en línea] <http://www.rae.es>, fecha de consulta: julio-agosto de 2018.

SÁNCHEZ LÓPEZ, C. (2002). «Las construcciones con se. Estado de la cuestión», en C. SÁNCHEZ LÓPEZ (ed.), Las construcciones con se. Madrid: Visor Libros, 13-163.

TALMY, L. (2000). Toward a Cognitive Semantics. Volume 1: Concept Structuring Systems. Cambridge: MIT.

VERHAGEN, A. (2007). «Construal and Perspectivization», en D. GEERAERTS y H. CUYCKENS (eds.), The Oxford Handbook of Cognitive Linguistics. Oxford: Oxford University Press, 48-81. 